\title{
CONSOANTES LÍQUIDAS: OCORRÊNCIA DE ESTRATÉGIAS DE REPARO EM DIFERENTES FAIXAS ETÁRIAS E GRAVIDADES DO DESVIO FONOLÓGICO
}

\author{
Liquid consonants: Occurrence of repair strategies in \\ different age groups and severity of phonological disorders
}

\author{
Fernanda Marafiga Wiethan ${ }^{(1)}$, Roberta Michelon Melo ${ }^{(2)}$, Helena Bolli Mota ${ }^{(3)}$
}

\section{RESUMO}

Objetivo: investigar a relação dos tipos de estratégias de reparo utilizadas nas consoantes líquidas do Português Brasileiro de acordo com a faixa etária e a gravidade do desvio fonológico. Método: participaram do estudo 120 crianças, sendo 41 do sexo feminino e 79 do masculino, com idades entre quatro e oito anos e onze meses. Todas as crianças empregavam pelo menos uma das estratégias envolvendo alguma das consoantes líquidas (/l/, / / /, / $/ /$, /R/) - substituição, semivocalização e/ou omissão - com emprego de $40 \%$ ou mais da estratégia no sistema fonológico. Os dados foram extraídos das avaliações fonológicas de crianças que não haviam recebido terapia fonológica, e a gravidade do desvio fonológico foi determinada por meio do Cálculo do Percentual de Consoantes Corretas - Revisado. Os dados foram submetidos à análise estatística por meio do Teste Exato de Fisher, com $\mathrm{p}<0.05$. Resultados: não houve diferença no emprego das estratégias de reparo utilizadas para a classe das líquidas conforme a faixa etária. Porém, foram observadas diferenças estatísticas na comparação entre a gravidade do desvio e estratégias de reparo das líquidas em relação a alguns dos segmentos e estratégias estudadas. Conclusão: conclui-se que a variável extralinguística, faixa etária, não está diretamente relacionada com a utilização de estratégias de reparo para as consoantes líquidas no desvio fonológico. No entanto, atenta-se para a influência da gravidade do desvio fonológico sobre o uso destas estratégias, que varia conforme o segmento-alvo.

DESCRITORES: Distúrbios da Fala; Patologia da Fala e Linguagem; Linguagem Infantil; Fala

\section{INTRODUÇÃO}

Inúmeros estudos tratam da aquisição dos fonemas pelas crianças com desenvolvimento fonológico normal e desviante, sendo que estes

(1) Fonoaudióloga; Mestranda em Distúrbios da Comunicação Humana pela Universidade Federal de Santa Maria (UFSM); Bolsista CAPES, Santa Maria, RS, Brasil.

(2) Fonoaudióloga; Mestranda em Distúrbios da Comunicação Humana pela Universidade Federal de Santa Maria (UFSM); Bolsista CAPES, Santa Maria, RS, Brasil.

(3) Fonoaudióloga; Professora Associada do Curso de Fonoaudiologia e do Programa de Pós-Graduação em Distúrbios da Comunicação Humana da Universidade Federal de Santa Maria (UFSM), Santa Maria, RS, Brasil; Doutora em Lingüística Aplicada pela Pontifícia Universidade Católica do Rio Grande do Sul.

Conflito de interesses: inexistente apontam que a aquisição da classe das líquidas, tanto na língua portuguesa quanto na língua inglesa, é a mais tardia devido à suas propriedades acústicas e articulatórias ${ }^{1-4}$.

As líquidas do Português Brasileiro (PB), em termos de sistema fonológico, são $/ / /, / \AA / /, / \mathrm{R} / \mathrm{e} / \mathrm{r} / 5,6$. A líquida /// é observada nas posições de onset (inicial e medial) e coda (medial e final), a qual, em geral, é produzida como o glide [w], em termos de estrutura silábica, este fonema pode compor o onset simples e complexo; o / $/$ / ocorre na posição de onset medial e em poucas palavras, na posição de onset inicial; o /R/ apresenta-se em onset inicial e medial e na posição de coda na maioria dos Estados brasileiros; $\mathrm{o} / \mathrm{r} /$ apresenta-se em onset simples (medial) e em onset complexo, além de ser produzido em coda medial e final na região sul do Brasil. 
Quanto ao modo articulatório, estes fonemas são produzidos com um obstáculo à passagem do ar que se restringe a um ponto, que subdivide a classe das líquidas em laterais $(/ / /$ e $/ \delta /$ ), quando o obstáculo à passagem aérea é mantido e o ar é então desviado pelos lados; e não-laterais ou vibrantes (/r/, /R/), em que o padrão articulatório é realizado com uma ou várias batidas da língua no palato ${ }^{7}$. Estas batidas da língua no palato, em inglês, são chamadas de "tap" ou "flap", sendo o $/ r /$ tanto para o inglês quanto para o PB, um som sonoro e alveolar, pois a lâmina da língua toca na região dos alvéolos ${ }^{8}$.

Com relação à aquisição desta classe em crianças com desenvolvimento normal, verificouse que as laterais surgem primeiro, seguidas das não-laterais, obedecendo à seguinte ordem: $/ / /$, $/ \AA /$, /R/ e $/ \mathrm{r} / 2$. Dentre as líquidas, o $/ / /$ é o fonema que apresenta maior índice de produção correta, sendo pouco omitido ou substituído por outras líquidas ou outros fonemas de outras classes de sons ${ }^{9,10}$. Por sua vez, o fonema $/ r$ / é considerado de aquisição mais tardia, por ser de difícil produção, sofrendo maior número de estratégias de reparo ${ }^{2,4,9,10}$.

Na língua inglesa também foi observada a aquisição tardia da classe das líquidas. Um estudo, objetivando estabelecer hierarquias de traços distintivos e verificar a influência da posição do fonema na sílaba, investigou a fala de 40 crianças com desenvolvimento típico e idade média de 25 meses, falantes do inglês americano. O mesmo evidenciou que a maioria das crianças não apresentava os fonemas $/ / /$ e $/ r /$ em seus inventários fonéticos. Além disso, os autores estabeleceram níveis de complexidade de traços, sendo que o nível IV, mais complexo da hierarquia, contém os traços [metátese retardada]; [lateral] e [estridente] ${ }^{11}$.

Em estudo sobre o uso de estratégias de reparo em 240 crianças de três a oito anos de idade com aquisição fonológica normal, verificou-se que aos três, quatro e cinco anos, as estratégias de redução de encontro consonantal, substituição de líquida não-lateral por lateral e apagamento de coda foram os mais utilizados, todos envolvendo as líquidas /// e $/ r / 12$. Outro estudo mostra que, em geral, a estratégia mais utilizada pelas crianças durante a aquisição é a substituição dos segmentos $/ / /, / \delta /$ e $/ r /$ pelo glide $/ y /$ no $\mathrm{PB}$, o que pode ser atribuído ao não reconhecimento de uma ou mais características acústicas que os adultos utilizam para distinguir estes sons ${ }^{13}$. Desta forma, os estudos que tratam desta classe de sons são muito importantes, especialmente quando relacionados ao desvio fonológico (DF), pois podem auxiliar na busca por estratégias terapêuticas mais bem-sucedidas.
Um estudo sobre a prevalência de alterações fonológicas em 1076 escolares com idades entre 7:0 e 7:11 mostrou que as cinco estratégias de reparo mais utilizados entre as crianças com DF são: Redução do Encontro Consonantal (60,67\%), Substituição de Líquidas (47,19\%), Dessonorização de Fricativas (22,47\%), Dessonorização de Plosivas $(19,1 \%)$ e Apagamento de coda $(11,53 \%)^{14}$. Três das cinco estratégias mencionados envolvem a presença das consoantes líquidas. Quanto às estratégias de reparo de estrutura silábica, dentre as estratégias de apagamento realizadas pelas crianças com DF, a redução de encontro consonantal e o apagamento de líquida não-lateral na posição de coda são os que apresentaram maior número de ocorrências ${ }^{15}$.

Em pesquisa sobre a aquisição fonológica de dois sujeitos submetidos à terapia fonoaudiológica, verificou-se que ocorreu descontinuidade no processo de aquisição para todos os fonemas, porém a nãolinearidade foi mais acentuada para a classe das líquidas, confirmando assim a maior complexidade destes segmentos, por ser necessária a especificação de um maior número de traços marcados ${ }^{5,16}$. Outro estudo, sobre terapia fonológica em quatro sujeitos com idades de 6:0 a 8:0, com DF, mostrou que, antes de iniciar o tratamento, todos os sujeitos apresentavam alterações na classe das líquidas, sendo que estas alterações foram as últimas a serem superadas com a terapia ${ }^{17}$.

Especificamente sobre os róticos, em estudo sobre a aquisição desviante destes segmentos, verificou-se que a variável sexo influencia no processo, ocorrendo mais alterações em meninos. Além disso, quando o / $\mathrm{r} /$ encontra-se em onset simples, há mais casos de substituição do que de omissão, já na posição de onset complexo e coda o fonema é mais omitido. As substituições mencionadas, em geral, envolviam fonemas da mesma classe natural, com baixa ocorrência de processos idiossincráticos ${ }^{18}$.

Alguns estudos relacionam a ocorrência de estratégias de reparo com fatores que não são diretamente relacionados ao DF como desnutrição ou histórico de otites. O primeiro, sobre o desenvolvimento fonológico de crianças com antecedentes de desnutrição, mostrou que crianças com idades entre 6:0 e 6:6 apresentaram o processo fonológico de simplificação de líquidas e redução de encontro consonantal, os quais já deveriam estar suprimidos em relação à idade dos sujeitos ${ }^{19}$. Resultados semeIhantes foram obtidos no estudo que comparou o desenvolvimento fonológico de crianças com DF com e sem histórico de otite, o qual evidenciou que o processo mais operante para o grupo com otite em prova de imitação foi a simplificação de líquidas 
e no grupo sem otite, em prova de nomeação, foi a redução de encontro consonantal ${ }^{20}$.

Os estudos abordados evidenciam que a classe das líquidas é de aquisição tardia, sendo os segmentos mais difíceis para produção, tanto do ponto de vista acústico quanto articulatório, sofrendo maior número de estratégias de reparo.

Desta forma, o objetivo do presente estudo foi investigar a relação dos tipos de estratégias de reparo utilizadas nas consoantes líquidas do PB de acordo com as variáveis faixa etária e gravidade do DF.

\section{MÉTODO}

Os dados deste estudo são provenientes do banco de dados de um projeto de pesquisa vinculado a uma instituição de ensino superior do país. Compõem o banco de dados deste projeto um total de 197 sujeitos, todos com diagnóstico de DF. Considerando-se os critérios de inclusão e exclusão adotados, o corpus da presente pesquisa constou dos dados de fala de 120 sujeitos, sendo 41 do sexo feminino e 79 do masculino, com idades entre quatro e oito anos e onze meses.

Para que os dados dos sujeitos fossem incluídos nesta pesquisa, os seguintes critérios foram considerados: estarem autorizados pelos pais ou responsáveis a participar da pesquisa por meio do Termo de Consentimento Livre e Esclarecido (TCLE), ter idades entre quatro e oito anos e onze meses e realizar a (s) estratégia (s) de substituição, semivocalização e/ou omissão de alguma das consoantes líquidas do PB (///, / $/ / /, / \mathrm{r} /$, /R/), com emprego de $40 \%$ ou mais da estratégia de reparo em seu sistema fonológico ${ }^{21}$.

Como critérios de exclusão adotaram-se os seguintes aspectos: sujeitos que tivessem recebido qualquer tipo de terapia fonoaudiológica anterior à primeira avaliação do sistema fonológico, a não assinatura do TCLE, presença de alterações fonoaudiológicas além do DF e presença de comprometimentos evidentes nas áreas neurológica, cognitiva e psicológica.

Foram analisados os dados de fala da primeira avaliação do sistema fonológico do sujeito, sendo, portanto, anterior ao início da terapia fonológica. O instrumento utilizado foi a Avaliação Fonológica da Criança $(\mathrm{AFC})^{22}$, após a transcrição fonética dos dados de fala, foi realizada a análise contrastiva. Todos os dados foram revistos e julgados separadamente por mais dois julgadores com experiência em transcrição fonética e análise perceptivo-auditiva.

A partir dos resultados desta análise foi determinada a gravidade do DF, por meio do Cálculo do Percentual de Consoantes Corretas - Revisado
(PCC-R $)^{23}$ que desconsidera as distorções produzidas pelos sujeitos e é baseado na classificação do Percentual de Consoantes Corretas (PCC) ${ }^{24}$, este então divide a gravidade do DF em Leve (DL), Moderado-Leve (DML), Moderado Grave (DMG) e Grave (DG). O PCC é calculado dividindo-se o número de consoantes produzidas corretamente pelo número de consoantes totais produzidas. Desta forma o DL tem PCC entre $86 \%$ e $100 \%$, o DML PCC entre 66 e 85\%, o DMG PCC entre 51 e $65 \%$ e o DG PCC menor que $50 \%$.

Foram analisadas as seguintes variáveis com relação à produção das consoantes líquidas (/l/, $/ \delta /, / r /, / R /)$ produzidas por crianças com DF: gravidade do DF, faixa etária e estratégia de reparo empregada para cada fonema (semivocalização de líquida, substituição de líquida, omissão de líquida, estratégia incomum e nenhuma estratégia). Quando um sujeito empregava mais de uma estratégia de reparo para o mesmo fonema, era estabelecida outra categoria, por exemplo, para o fonema $/ r /$, semivocalização/omissão. Para a análise das estratégias de reparo foi considerado o sistema fonológico geral, estando todos os fonemas na posição de onset - inicial e medial.

O projeto de pesquisa, ao qual o presente estudo está vinculado, foi devidamente aprovado pelo Comitê de Ética e Pesquisa da Universidade Federal de Santa Maria sob o número 052/04.

Os dados do estudo foram submetidos à análise estatística por meio do programa Statistical Analysis System, versão 8.02, utilizando-se o Teste Exato de Fisher. O nível de significância adotado para os testes estatísticos foi de $5 \%(p<0.05)$.

\section{RESULTADOS}

As Tabelas 1 a 4 apresentam as comparações da variável extralinguística faixa etária com as estratégias de reparo apresentadas pelos sujeitos para cada um dos fonemas estudados. As faixas etárias de 7:0 a 7:11 e de 8:0 a 8:11 foram agrupadas para maior consistência nas análises, devido à baixa frequência de crianças nesta última faixa. Para os fonemas /I/, / / / e /R/, observou-se predomínio de não ocorrência do emprego de estratégias de reparo para todas as faixas etárias. Já para o fonema/r/, houve predomínio da estratégia de substituição de líquida, também para todas as faixas etárias. Os dados apresentados apontam resultados estatisticamente equilibrados, portanto não há diferença no emprego das estratégias de reparo utilizadas para a classe das líquidas em relação à variável faixa etária. 
Tabela 1 - Comparação entre faixa etária e estratégias empregadas para o fonema /I/

\begin{tabular}{|c|c|c|c|c|c|c|}
\hline Estratégias & & $4: 0-4: 11$ & $5: 0-5: 11$ & $6: 0-6: 11$ & $7: 0-8: 11$ & Valor de $p$ \\
\hline \multirow[t]{2}{*}{ Nenhuma } & $\mathrm{n}$ & 18 & 30 & 23 & 9 & \multirow{12}{*}{$p=0.548$} \\
\hline & $\%$ & 62.0 & 66.7 & 69.7 & 69.2 & \\
\hline \multirow[t]{2}{*}{ Semivocalização } & $\mathrm{n}$ & 2 & 4 & 2 & 1 & \\
\hline & $\%$ & 6.9 & 8.9 & 6.1 & 7.7 & \\
\hline \multirow[t]{2}{*}{ Substituição } & $\mathrm{n}$ & 0 & 1 & 0 & 1 & \\
\hline & $\%$ & 0.0 & 2.2 & 0.0 & 7.7 & \\
\hline \multirow[t]{2}{*}{ Omissão } & $\mathrm{n}$ & 5 & 8 & 6 & 0 & \\
\hline & $\%$ & 17.2 & 17.8 & 18.2 & 0.0 & \\
\hline \multirow[t]{2}{*}{ Incomum } & $\mathrm{n}$ & 0 & 0 & 1 & 0 & \\
\hline & $\%$ & 0.0 & 0.0 & 3.0 & 0.0 & \\
\hline \multirow[t]{2}{*}{ Sem./Om. } & $\mathrm{n}$ & 4 & 2 & 1 & 2 & \\
\hline & $\%$ & 13.7 & 4.4 & 3.0 & 15.4 & \\
\hline
\end{tabular}

Legenda 1: n: número de sujeitos; Sem./Om.: semivocalização/omissão. Teste estatístico utilizado: Teste Exato de Fisher, com $\mathrm{p}<0,05$.

Tabela 2 - Comparação entre faixa etária e estratégias empregadas para o fonema /rl

\begin{tabular}{|c|c|c|c|c|c|c|}
\hline Estratégias & & $4: 0-4: 11$ & $5: 0-5: 11$ & $6: 0-6: 11$ & $7: 0-8: 11$ & Valor de $\mathrm{p}$ \\
\hline \multirow[t]{2}{*}{ Nenhuma } & $\mathrm{n}$ & 4 & 7 & 1 & 4 & \multirow{14}{*}{$p=0.214$} \\
\hline & $\%$ & 13.8 & 15.6 & 3.0 & 30.8 & \\
\hline \multirow[t]{2}{*}{ Semivocalização } & $\mathrm{n}$ & 4 & 8 & 7 & 2 & \\
\hline & $\%$ & 13.8 & 17.8 & 21.2 & 15.4 & \\
\hline \multirow[t]{2}{*}{ Substituição } & $\mathrm{n}$ & 11 & 14 & 10 & 4 & \\
\hline & $\%$ & 38.0 & 31.1 & 30.3 & 30.7 & \\
\hline \multirow[t]{2}{*}{ Omissão } & $\mathrm{n}$ & 3 & 9 & 10 & 3 & \\
\hline & $\%$ & 10.3 & 20.0 & 30.3 & 23.1 & \\
\hline \multirow[t]{2}{*}{ Incomum } & $\mathrm{n}$ & 0 & 0 & 3 & 0 & \\
\hline & $\%$ & 0.0 & 0.0 & 9.1 & 0.0 & \\
\hline \multirow[t]{2}{*}{ Sem./Om. } & $\mathrm{n}$ & 6 & 6 & 2 & 0 & \\
\hline & $\%$ & 20.7 & 13.3 & 6.1 & 0.0 & \\
\hline \multirow[t]{2}{*}{ Subs./Om. } & $\mathrm{n}$ & 1 & 1 & 0 & 0 & \\
\hline & $\%$ & 3.4 & 2.2 & 0.0 & 0.0 & \\
\hline
\end{tabular}


Tabela 3 - Comparação entre faixa etária e estratégias empregadas para o fonema / $\mathrm{A}$ /

\begin{tabular}{|c|c|c|c|c|c|c|}
\hline Estratégias & & $4: 0-4: 11$ & $5: 0-5: 11$ & $6: 0-6: 11$ & $7: 0-8: 11$ & Valor de $p$ \\
\hline \multirow[t]{2}{*}{ Nenhuma } & $\mathrm{n}$ & 8 & 17 & 16 & 4 & \multirow{14}{*}{$p=0.236$} \\
\hline & $\%$ & 27.6 & 37.8 & 48.5 & 30.8 & \\
\hline \multirow[t]{2}{*}{ Semivocalização } & $\mathrm{n}$ & 12 & 12 & 11 & 6 & \\
\hline & $\%$ & 41.4 & 26.7 & 33.3 & 46.1 & \\
\hline \multirow[t]{2}{*}{ Substituição } & $\mathrm{n}$ & 7 & 12 & 3 & 3 & \\
\hline & $\%$ & 24.1 & 26.7 & 9.1 & 23.1 & \\
\hline \multirow[t]{2}{*}{ Omissão } & $\mathrm{n}$ & 1 & 2 & 0 & 0 & \\
\hline & $\%$ & 3.4 & 4.4 & 0.0 & 0.0 & \\
\hline \multirow[t]{2}{*}{ Incomum } & $\mathrm{n}$ & 0 & 0 & 3 & 0 & \\
\hline & $\%$ & 0.0 & 0.0 & 9.1 & 0.0 & \\
\hline \multirow[t]{2}{*}{ Sem./Om. } & $\mathrm{n}$ & 0 & 2 & 0 & 0 & \\
\hline & $\%$ & 0 & 4.4 & 0.0 & 0.0 & \\
\hline \multirow[t]{2}{*}{ Subs./Sem. } & $\mathrm{n}$ & 1 & 0 & 0 & 0 & \\
\hline & $\%$ & 3.4 & 0.0 & 0.0 & 0.0 & \\
\hline
\end{tabular}

Legenda 3: n: número de sujeitos; Sem./Om.: semivocalização/omissão; Subs./Sem.: substituição/semivocalização. Teste estatístico utilizado: Teste Exato de Fisher, com $\mathrm{p}<0,05$.

Tabela 4 - Comparação entre faixa etária e estratégias empregadas para o fonema /R/

\begin{tabular}{|c|c|c|c|c|c|c|}
\hline Estratégias & & $4: 0-4: 11$ & $5: 0-5: 11$ & $6: 0-6: 11$ & $7: 0-8: 11$ & Valor de $p$ \\
\hline \multirow[t]{2}{*}{ Nenhuma } & $\mathrm{n}$ & 14 & 27 & 27 & 10 & \multirow{10}{*}{$p=0.221$} \\
\hline & $\%$ & 48.3 & 60.0 & 81.8 & 76.9 & \\
\hline \multirow[t]{2}{*}{ Semivocalização } & $\mathrm{n}$ & 1 & 1 & 1 & 0 & \\
\hline & $\%$ & 3.4 & 2.2 & 3.0 & 0.0 & \\
\hline \multirow[t]{2}{*}{ Substituição } & $\mathrm{n}$ & 2 & 0 & 0 & 0 & \\
\hline & $\%$ & 6.9 & 0.0 & 0.0 & 0.0 & \\
\hline \multirow[t]{2}{*}{ Omissão } & $\mathrm{n}$ & 10 & 13 & 4 & 2 & \\
\hline & $\%$ & 34.5 & 28.9 & 12.1 & 15.4 & \\
\hline \multirow[t]{2}{*}{ Sem./Om. } & $\mathrm{n}$ & 2 & 4 & 1 & 1 & \\
\hline & $\%$ & 6.9 & 8.9 & 3.0 & 7.7 & \\
\hline
\end{tabular}

Legenda 4: n: número de sujeitos; Sem./Om.: semivocalização/omissão. Teste estatístico utilizado: Teste Exato de Fisher, com $\mathrm{p}<0,05$.

As Tabelas 5 a 8 apresentam as comparações entre a variável gravidade do DF e as estratégias de reparo apresentadas pelos sujeitos para cada um dos fonemas investigados. Verificou-se diferença estatisticamente significativa nas seguintes comparações:

- Para o fonema ///: maior frequência da estratégia de reparo de omissão de líquida para os graus DML, DMG e DG, semivocalização de líquida para o grau DML, semivocalização/omissão de líquida para o grau DG e, a não ocorrência de emprego de estratégias para o grau DL (Tabela 5);

- Para o fonema $/ \hbar /$ : maior frequência da estratégia de semivocalização de líquida para os graus DML, DMG e DG, substituição de líquida para os graus DML e DMG e, a não ocorrência de emprego de estratégias para o grau DL (Tabela 7);

- Para o fonema /R/: maior frequência da estratégia de omissão para os graus DML, DMG e DG, semivocalização/omissão de líquida para o grau DG, e também, a não ocorrência de emprego de estratégias para o grau DL (Tabela 8).

Porém, para o fonema $/ r /$, não foi observada diferença estatística em nenhuma das relações estabelecidas (Tabela 6). 
Tabela 5 - Comparação entre a gravidade do desvio fonológico e estratégias empregadas para o fonema /I/

\begin{tabular}{|c|c|c|c|c|c|c|}
\hline Estratégias & & $D L$ & $D M L$ & $D M G$ & $D G$ & Valor de $\mathrm{p}$ \\
\hline \multirow[t]{2}{*}{ Nenhuma } & $\mathrm{n}$ & 17 & 44 & 16 & 3 & \multirow{12}{*}{$p<0,001^{*}$} \\
\hline & $\%$ & $100.0^{*}$ & 69.8 & 61.5 & 21.4 & \\
\hline \multirow[t]{2}{*}{ Semivocalização } & $\mathrm{n}$ & 0 & 6 & 2 & 1 & \\
\hline & $\%$ & 0.0 & $9.5^{\star}$ & 7.7 & 7.1 & \\
\hline \multirow[t]{2}{*}{ Substituição } & $n$ & 0 & 2 & 0 & 0 & \\
\hline & $\%$ & 0.0 & 3.2 & 0.0 & 0.0 & \\
\hline \multirow[t]{2}{*}{ Omissão } & $\mathrm{n}$ & 0 & 7 & 6 & 6 & \\
\hline & $\%$ & 0.0 & $11.1^{*}$ & $23.1^{*}$ & $42.8^{*}$ & \\
\hline \multirow[t]{2}{*}{ Incomum } & $\mathrm{n}$ & 0 & 0 & 1 & 0 & \\
\hline & $\%$ & 0.0 & 0.0 & 3.8 & 0.0 & \\
\hline \multirow[t]{2}{*}{ Sem./Om. } & $\mathrm{n}$ & 0 & 4 & 1 & 4 & \\
\hline & $\%$ & 0.0 & 6.3 & 3.8 & $28.6^{\star}$ & \\
\hline
\end{tabular}

Legenda 5: n: número de sujeitos; Sem./Om.: semivocalização/omissão; DL: Desvio Leve; DML: Desvio Moderado Leve; DMG: Desvio Moderado Grave; DG: Desvio Grave. *Resultados em que houve significância estatística. Teste estatístico utilizado: Teste Exato de Fisher, com $\mathrm{p}<0,05$.

Tabela 6 - Comparação entre a gravidade do desvio fonológico e estratégias empregadas para o fonema $/ r /$

\begin{tabular}{|c|c|c|c|c|c|c|}
\hline Estratégias & & $D L$ & $D M L$ & $D M G$ & $D G$ & Valor de $\mathrm{p}$ \\
\hline \multirow[t]{2}{*}{ Nenhuma } & $n$ & 2 & 11 & 3 & 0 & \multirow{14}{*}{$p=0,375$} \\
\hline & $\%$ & 11.76 & 17.5 & 11.5 & 0.0 & \\
\hline \multirow[t]{2}{*}{ Semivocalização } & $\mathrm{n}$ & 9 & 10 & 5 & 4 & \\
\hline & $\%$ & 52.9 & 15.9 & 19.2 & 28.6 & \\
\hline \multirow[t]{2}{*}{ Substituição } & $\mathrm{n}$ & 4 & 20 & 7 & 3 & \\
\hline & $\%$ & 23.5 & 31.7 & 26.9 & 21.4 & \\
\hline \multirow[t]{2}{*}{ Omissão } & $\mathrm{n}$ & 0 & 14 & 5 & 2 & \\
\hline & $\%$ & 0.0 & 22.2 & 19.2 & 14.3 & \\
\hline \multirow[t]{2}{*}{ Incomum } & $\mathrm{n}$ & 0 & 2 & 1 & 0 & \\
\hline & $\%$ & 0.0 & 3.2 & 3.8 & 0.0 & \\
\hline \multirow[t]{2}{*}{ Sem./Om. } & $\mathrm{n}$ & 0 & 6 & 4 & 4 & \\
\hline & $\%$ & 0.0 & 9.5 & 15.4 & 28.9 & \\
\hline \multirow[t]{2}{*}{ Sem./Subs. } & $\mathrm{n}$ & 0 & 0 & 1 & 1 & \\
\hline & $\%$ & 0.0 & 0.0 & 3.8 & 7.14 & \\
\hline
\end{tabular}

Legenda 6: n: número de sujeitos; Sem./Om.: semivocalização/omissão; Sem./Subs.: semivocalização/substituição; DL: Desvio Leve; DML: Desvio Moderado Leve; DMG: Desvio Moderado Grave; DG: Desvio Grave. Teste estatístico utilizado: Teste Exato de Fisher, com $\mathrm{p}<0,05$. 
Tabela 7 - Comparação entre a gravidade do desvio fonológico e estratégias empregadas para o fonema / $\lambda$ /

\begin{tabular}{|c|c|c|c|c|c|c|}
\hline Estratégias & & $D L$ & $D M L$ & $D M G$ & $D G$ & Valor de $p$ \\
\hline \multirow[t]{2}{*}{ Nenhuma } & $\mathrm{n}$ & 13 & 24 & 5 & 3 & \multirow{14}{*}{$p=0.021^{*}$} \\
\hline & $\%$ & $76.5^{\star}$ & 38.1 & 19.2 & 21.4 & \\
\hline \multirow[t]{2}{*}{ Semivocalização } & $\mathrm{n}$ & 2 & 22 & 10 & 7 & \\
\hline & $\%$ & 11.8 & $34.9^{*}$ & $38.46^{*}$ & $50.0^{*}$ & \\
\hline \multirow[t]{2}{*}{ Substituição } & $\mathrm{n}$ & 2 & 12 & 9 & 2 & \\
\hline & $\%$ & 11.8 & $19.0^{*}$ & $34.6^{\star}$ & 14.3 & \\
\hline \multirow[t]{2}{*}{ Omissão } & $\mathrm{n}$ & 0 & 2 & 1 & 0 & \\
\hline & $\%$ & 0.0 & 3.17 & 3.8 & 0.0 & \\
\hline \multirow[t]{2}{*}{ Incomum } & $\mathrm{n}$ & 0 & 2 & 1 & 0 & \\
\hline & $\%$ & 0.0 & 3.17 & 3.8 & 0.0 & \\
\hline \multirow[t]{2}{*}{ Sem./Om. } & $\mathrm{n}$ & 0 & 0 & 0 & 2 & \\
\hline & $\%$ & 0.0 & 0.0 & 0.0 & 14.3 & \\
\hline \multirow[t]{2}{*}{ Sem./Subs. } & $\mathrm{n}$ & 0 & 1 & 0 & 0 & \\
\hline & $\%$ & 0.0 & 1.6 & 0.0 & 0.0 & \\
\hline
\end{tabular}

Legenda 7: n: número de sujeitos; Sem./Om.: semivocalização/omissão; Sem./Subs.: semivocalização/substituição; DL: Desvio Leve; DML: Desvio Moderado Leve; DMG: Desvio Moderado Grave; DG: Desvio Grave. *Resultados em que houve significância estatística. Teste estatístico utilizado: Teste Exato de Fisher, com $\mathrm{p}<0,05$.

Tabela 8 - Comparação entre a gravidade do desvio fonológico e estratégias empregadas para o fonema /R/

\begin{tabular}{|c|c|c|c|c|c|c|}
\hline Estratégias & & $D L$ & $D M L$ & $D M G$ & $D G$ & Valor de $\mathrm{p}$ \\
\hline \multirow[t]{2}{*}{ Nenhuma } & $\mathrm{n}$ & 17 & 44 & 11 & 6 & \multirow{10}{*}{$p<0,01^{*}$} \\
\hline & $\%$ & $100.0^{*}$ & 69.8 & 42.3 & 42.9 & \\
\hline \multirow[t]{2}{*}{ Semivocalização } & $\mathrm{n}$ & 0 & 2 & 1 & 0 & \\
\hline & $\%$ & 0.0 & 3.2 & 3.8 & 0.0 & \\
\hline \multirow[t]{2}{*}{ Substituição } & $\mathrm{n}$ & 0 & 2 & 0 & 0 & \\
\hline & $\%$ & 0.0 & 3.2 & 0.0 & 0.0 & \\
\hline \multirow[t]{2}{*}{ Omissão } & $\mathrm{n}$ & 0 & 13 & 12 & 4 & \\
\hline & $\%$ & 0.0 & $20.6^{*}$ & $46.1^{\star}$ & $28.6^{*}$ & \\
\hline \multirow[t]{2}{*}{ Sem./Om. } & $\mathrm{n}$ & 0 & 2 & 2 & 4 & \\
\hline & $\%$ & 0.0 & 3.2 & 7.7 & $28.6^{*}$ & \\
\hline
\end{tabular}

Legenda 8: n: número de sujeitos; Sem./Om.: semivocalização/omissão; Sem./Subs.: semivocalização/substituição; DL: Desvio Leve; DML: Desvio Moderado Leve; DMG: Desvio Moderado Grave; DG: Desvio Grave. Teste estatístico utilizado: Teste Exato de Fisher, com $p<0,05$.

\section{DISCUSSÃO}

O predomínio de não ocorrência do emprego de estratégias de reparo para os fonemas $/ / /, / \hbar /$ e /R/ em todas as faixas etárias pode ser explicado pelo fato de, na classe das líquidas, estes fonemas serem adquiridos antes que $0 / r^{2}$. Além disso, estudos apontam que o fonema /// é o que apresenta maior índice de produções corretas, sendo acometido por poucas estratégias de reparo ${ }^{9,10}$.

Com relação aos resultados observados para o fonema $/ r /$, não se verificou diferença estatística entre as faixas etárias, observando-se predomínio da estratégia de substituição de líquidas para todas as idades analisadas. Vários estudos apontaram maior ocorrência de estratégias de reparo para o 
fonema /r/ por este ser de aquisição mais tardia no $\mathrm{PB}^{2,4,9-10}$ e em outras línguas, como no inglês ${ }^{25}$.

Além disso, os fonemas róticos encontram-se localizados no nível de maior complexidade por apresentarem traços que são especificados mais tarde no desenvolvimento fonológico, por isso sofrem maior número de estratégias de reparo. Sabe-se ainda que, na hierarquia de traços, um traço mais complexo ou marcado implica, necessariamente, a ocorrência de um traço menos complexo ou não-marcado (mas não o contrário). Este fato pode explicar a maior ocorrência de alterações na líquida não-lateral /r/5,26.

Em relação à comparação entre os graus de DF verificou-se predomínio de não ocorrência de estratégias de reparo para os fonemas $/ / /, / K / \mathrm{e} / \mathrm{R} /$ para o DL. Este resultado sustenta os dados de aquisição fonológica, tanto normal quanto desviante, uma vez que, na classe das líquidas, estes fonemas emergem antes da líquida não-lateral /r/. Nos casos de DL é observada uma fonologia mais rica, na qual existem poucas estratégias de reparo atuantes no sistema fonológico ${ }^{27}$.

De maneira oposta, as crianças que apresentam DG mostram menor conhecimento fonológico e apresentam maior número de estratégias de reparo, como observado nos resultados desta pesquisa, em que este grau foi o único a apresentar associação de mais de uma estratégia empregada para o mesmo fonema, no caso /// e /R/, com significância estatística.

Outro dado que evidencia um pobre conhecimento fonológico é o uso da estratégia de reparo de omissão de segmento, onde perceptivo-auditivamente, o sujeito parece simplesmente omitir o segmento ao invés de utilizar outro, próximo ao som-alvo, como no caso de estratégias de substituição de líquida ou assimilação, que denotam um conhecimento subjacente do falante em relação à produção dos segmentos e/ou estruturas fonológicas $^{2}$. Por esta razão não foi observada esta estratégia para nenhum dos fonemas estudados no grau de desvio menos acentuado (DL).

Na literatura, em geral, verifica-se alta ocorrência da estratégia de semivocalização, a qual ocorre por esta ser uma etapa que faz parte da cronologia da aquisição das líquidas, devido à complexidade articulatória e acústica dessas consoantes ${ }^{13,28}$. No presente estudo verificou-se significância estatística para a ocorrência desta estratégia nos graus DML para o fonema /// e DML, DMG e DG para o fonema $/ \hbar /$.
Em uma pesquisa que objetivou descrever o desenvolvimento fonológico de crianças em idade pré-escolar, falantes da variação mineira do PB, foi observado que $25 \%$ da amostra fazia uso de estratégias de reparo envolvendo apenas a classe das líquidas. Em sua amostra geral, os autores verificaram ainda que a estratégia de reparo mais frequente era a de substituição destas consoantes ${ }^{29}$. Outra pesquisa também menciona a alta ocorrência desta estratégia para todas as consoantes líquidas ${ }^{14}$. No presente estudo foi verificado por meio de resultados estatisticamente significantes, que a estratégia de substituição de líquida, foi significativamente empregada para o fonema $/ \AA /$ nos graus DML e DMG.

Quanto ao fonema / $\mathrm{r} /$, os resultados mostraramse estatisticamente equilibrados, o que é justificado pela aquisição tardia deste fonema, o qual é acometido por um maior número de estratégias de reparo em todas as gravidades do $\mathrm{DF}^{2,4,9,10}$. Desta forma, observou-se mais de uma estratégia para todos os graus do desvio.

Embora não tenha mostrado significância estatística, é interessante mencionar a baixa frequência de estratégias incomuns ou idiossincráticas para todos os fonemas estudados, tanto em relação à faixa etária quanto em relação à gravidade do DF. Indo ao encontro da presente pesquisa, outro trabalho, que estudou a aquisição desviante das líquidas não-laterais, também verificou poucas ocorrências de estratégias incomuns ${ }^{18}$.

Sugere-se novas pesquisas na área de aquisição fonológica desviante, especialmente buscando utilizar amostras homogêneas entre os grupos estudados, a fim de proporcionar maior conhecimento à clínica fonoaudiológica frente ao diagnóstico do DF e à intervenção terapêutica destes casos.

\section{CONCLUSÃO}

Com este estudo verificou-se que a variável extralinguística, faixa etária, não está diretamente relacionada com a utilização de estratégias de reparo para as consoantes líquidas por crianças com DF, falantes do PB. No entanto, deve-se atentar para a influência da gravidade do DF sobre o uso destas estratégias, que varia conforme o segmentoalvo. Com isso, por meio da análise das estratégias de reparo, é possível inferir sobre as características do sistema fonológico de cada criança. 


\section{ABSTRACT}

Purpose: to investigate the relationship of the types of repair strategies used in the liquid consonants of Brazilian Portuguese according to age and severity of the phonological disorders. Method: the study included 120 children, 41 females and 79 males, aged between four and eight years and eleven months. All children used at least one of the strategies involving some of the liquid consonants $(/ / /$, / $\kappa /, / \mathrm{r} /, / \mathrm{R} /$ ) - replacement, semi-vocalization and / or omissions - using $40 \%$ or more of the strategy in the phonological system. Data were extracted from the phonological assessments of children who had not received phonological therapy, and the severity of phonological disorders was determined by the Percentage of Consonants Correct-Revised (PCC-R). Data were statistically analyzed by using Fisher's Exact Test, with $p<0.05$. Results: there was no difference as for the use of repair strategies for the liquid consonants based on age. However, statistical differences were observed when comparing the severity of the deviation and repair strategies of liquid consonants for some of the studied segments and strategies. Conclusion: we concluded that the extra-linguistic variable and age are not directly related to the use of repair strategies for the liquid consonants in phonological disorders. Nonetheless, we are aware on the influence of severity concerning phonological disorders on the use of these strategies, which varies according to the target segment.

\section{KEYWORDS: Speech Disorders; Speech-Language Pathology; Child Language; Speech}

\section{REFERÊNCIAS}

1. Hodson BW, Paden EP. Targeting intelligible speech: A phonological approach to remediation. 2nd ed. Austin: ProEd, 1991.

2. Lamprecht RR. A aquisição da fonologia do português na faixa etária dos 2:9 - 5:5. Letras de Hoje. 1993; 28(2):99-106.

3. Mezzomo CL. Aquisição da coda no português brasileiro: uma análise via teoria de Princípios e Parâmetros [tese]. Porto Alegre (RS): Pontifícia Universidade Católica do Rio Grande do Sul; 2004.

4. Checalin MA. Estudo do Papel do Contexto Facilitador, segundo diferentes abordagens teóricas, na aquisição do r-fraco por crianças com desvio fonológico [Dissertação]. Santa Maria (RS): Universidade Federal de Santa Maria; 2008.

5. Mota HB. Aquisição segmental do português: um modelo implicacional de complexidade de traços [Tese]. Porto Alegre (RS): Pontifícia Universidade Católica do Rio Grande do Sul; 1996.

6. Hernandorena CLM, Lamprecht RR. A aquisição das consoantes líquidas do português. Letras Hoje. 1997; 32(4):7-22.

7. Russo IP, Behlau M. As pistas acústicas das vogais e consoantes. In:__. Percepção da fala: análise acústica. São Paulo: Lovise, 1993. p. 25-50.

8. Carr P. English Phonetics and Phonology: an introduction. Oxford: Blackwell, 1999.

9. Castro MM. Estudo da estimulabilidade de crianças com desenvolvimento típico e com distúrbio fonológico para os fonemas líquidos laterais e vibrante simples [mestrado]. São Paulo (SP): Faculdade de Filosofia Letras e Ciências Humanas da Universidade de São Paulo; 2004.

10. Wertzner H, Pagan-Neves I, Castro MM. Análise acústica e índice de estimulabilidade nos sons líquidos do português brasileiro. Rev CEFAC. 2007; 9(3): 339-50.

11. Stokes SF, Klee T, Carson CP, Carson D. A Phonemic Implicational Feature Hierarchy of Phonological Contrasts for English-Speaking Children. J Speech Lang Hear Res. 2005; 48: 817-33

12. Ferrante $C$, Van Borsel J, Pereira MMB. Análise dos processos fonológicos em crianças com desenvolvimento fonológico normal. Rev. Soc. Bras. Fonoaudiol. 2009; 14(1): 36-40.

13. Pagan LO, Wertzner HF. Análise acústica das consoantes líquidas do Português Brasileiro em crianças com e sem transtorno fonológico. Rev. Soc. Bras. Fonoaudiol. 2007; 12(2): 106-13.

14. Patah LK, Takiushi N. Prevalência das alterações fonológicas e uso dos processos fonológicos em escolares aos 7 anos. Rev CEFAC. 2008; 10(2): 158-67.

15. Grüdtner ACR. Os Processos fonológicos de estrutura silábica no desenvolvimento fonológico normal e nos desvios fonológicos evolutivos. V Congresso Brasileiro de Lingüística Aplicada; 1998; Porto Alegre: UFRGS; 1998.

16. Keske-Soares M, Pagliarin KC, Ghisleni MRL, Lamprecht RR. Aquisição não-linear durante o processo terapêutico. Letras de Hoje. 2008; 43(3): 22-6. 
17. Spindola RA, Payão LMC, Bandini HHM. Abordagem fonoaudiológica em desvios fonológicos fundamentada na hierarquia dos traços distintivos e na consciência fonológica. Rev. CEFAC. 2007; 9(2): 180-9.

18. Vidor D. Aquisição das líquidas não-laterais por crianças com desvios fonológicos evolutivos: descrição, análise e comparação com o desenvolvimento normal. Letras de Hoje. 2001; 36(3): 715-20.

19. Lima LM, Queiroga BAM. Aquisição fonológica em crianças com antecedentes de desnutrição. Rev. CEFAC. 2007; 9(1): 13-20.

20. Wertzner H, Pagan L, Galea D, Papp A. Características fonológicas de crianças com transtorno fonológico com e sem histórico de otite média. Rev. Soc. Bras. Fonoaudiol. 2007;12:41-7.

21. Bernhardt B. Developmental implications of nonlinear phonological theory. Clin Linguist Phon. 1992; 6(4):259-81.

22. Yavas M, Hernandorena CLM, Lamprecht RR. Avaliação fonológica da criança: reeducação e terapia. Porto Alegre: Artes Médicas; 1991.
23. Shriberg LD, Austin D, Lewis BA, Mcsweeny JL, Wilson DL. The percentage of consonants correct (PCC) metric: extensions and reliability data. J Speech Lang Hear Res. 1997; 40(4): 708-22.

24. Shriberg LD, Kwiatkowski J. Phonological disorders I: A Diagnostic classification system. J Speech Hear Dis. 1982; 47(1): 226-41.

25. Adler-Bock M, Bernhardt BM, Gick B, Bacsfalvi $P$. The Use of Ultrasound in Remediation of North American English / $r$ / in 2 Adolescents. Am J Speech Lang Pathol. 2007; 16: 128-39.

26. Gierut J. Phonological Complexity and Language Learnability. 2007; 16: 6-17.

27. Ribas LP. Aquisição das líquidas por crianças com desvio fonológico: Aquisição silábica ou segmental?. Letras (UFSM). 2008; 36: 129-49.

28. Cruz GFA. O processo de semivocalização de líquidas laterais em posição pré-vocálica: uma revisão teórica. Letrônica. 2009; 2 (2): 48-57.

29. Vitor RM, Cardoso-Martins C. Desenvolvimento fonológico de crianças pré-escolares da região noroeste de Belo Horizonte. Psicol. Rev. 2007; 13(2): 383-98.
http://dx.doi.org/10.1590/S1516-18462010005000141

RECEBIDO EM: 23/03/2010

ACEITO EM: 21/05/2010

Endereco para correspondência:

Fernanda Marafiga Wiethan.

Rua Júlio Nogueira, $n^{\circ} 130$, Uglione

Santa Maria, RS

CEP: 97070-510

E-mail: fernanda_wiethan@yahoo.com.br 